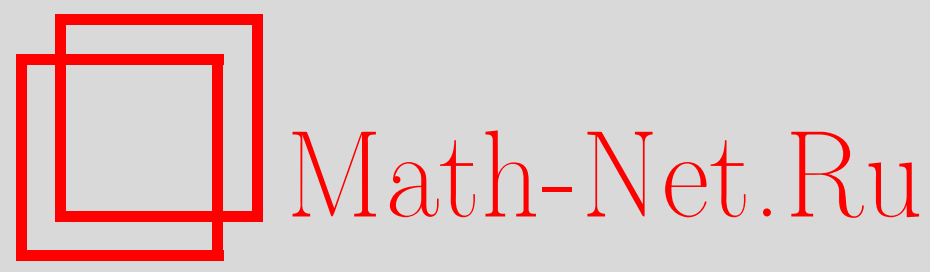

К. И. Пономарев, Об одной статистической модели стеганографии, Дискрет. матем., 2009, том 21, выпуск 2, 138-145

DOI: https://doi.org/10.4213/dm1053

Использование Общероссийского математического портала Math-Net.Ru подразумевает, что вы прочитали и согласны с пользовательским соглашением http://www.mathnet.ru/rus/agreement

Параметры загрузки:

IP : 54.198 .55 .26

26 апреля 2023 г., 14:58:18 


\title{
Об одной статистической модели стеганографии
}

\author{
() 2009 г. К. И. Пономарев
}

\begin{abstract}
Рассматривается статистическая модель, относящаяся к новому быстро развивающемуся направлению в сфере защиты информации, к так называемой стеганографии. Предлагается один из возможных вариантов скрытого вкрапления передаваемой информации в мультимедийные файлы и проводится ее вероятностно-статистический анализ.
\end{abstract}

\section{1. Введение}

Задача надежной защиты авторских прав, прав интеллектуальной собственности и других конфиденциальных данных от несанкционированного доступа является одной из старейших и не до конца решенных до настоящего времени проблем. Поэтому во всем мире постоянно ищут решение вопроса разработки методов защиты информации, среди них методы криптографии и стеганографии.

Криптографическая защита информации не снимает упомянутую выше проблему полностью, поскольку наличие шифрованного сообщения само по себе привлекает внимание противника, и он, завладев криптографически защищенным файлом и зная о размещении в нем секретной информации, направит все свои возможности на дешифрование секретных данных.

В настоящее время, в связи с развитием вычислительной техники и организации новых каналов передачи информации, появились новые, стенографические методы, в основе которых лежат особенности представления информации в компьютерных файлах, вычислительных сетях и т. П. Это дает возможность говорить о становлении нового быстро развивающегося направления в сфере защиты информации, компьютерной стеганографии, связанного как с цифровой обработкой сигналов, так и не связанного с таковой. В первом случае секретные сообщения встраиваются в цифровые данные, которые, как правило, имеют аналоговую природу - речь, изображения, аудио- и видеозаписи. Во втором случае конфиденциальная информация размещается в заголовках файлов различных форматов и в текстовых сообщениях. Подавляющее большинство текущих исследований в сфере стеганографии так или иначе связано именно с цифровой обработкой сигналов, что позволяет говорить о цифровой стеганографии.

Компьютерная стеганография активно использует как особенности программного обеспечения, протоколов для скрытия информации, так и приемы, использующие представление информации, предназначенные для восприятия органами зрения и слуха, в мультимедийном виде.

Анализ тенденций развития компьютерной стеганографии показывает, что в ближайшие годы интерес к ее развитию будет расти. Общеизвестно, что актуальность проблемы 
информационной безопасности постоянно растет и стимулирует поиск новых методов защиты информации. Сильным катализатором этого процесса является лавинообразное развитие компьютерной сети общего пользования, Интернета, и актуальность таких проблем, как защита авторских прав, прав на интеллектуальную собственность, организация электронной торговли, противоправная деятельность хакеров, террористов и т. п.

Как бы ни отличались направления использования стеганографии, выдвигаемые при этом требования во многом остаются неизменными. Так, исходные данные должны быть изменены так, чтобы эти изменения невозможно было выявить при визуальном контроле. Это требование определяет качество скрытия внедряемого сообщения, обеспечивая беспрепятственное прохождение стегосообщения по каналу связи так, чтобы оно никоим образом не могло привлечь внимание противника [1].

В то же время следует отметить, что математические, а конкретнее, теоретико-вероятностные и статистические вопросы стеганографии в настоящее время являются еще недостаточно проработанными, поэтому построение и анализ адекватных математических моделей представляет собой актуальную проблематику в этой области.

В данной работе предлагается один из возможных вариантов модели скрытого вкрапления передаваемой закрытой информации в мультимедийные файлы и проводится ее вероятностно-статистический анализ.

\section{2. Описание модели вкрапления}

Пусть имеется массив случайных двоичных знаков $\bar{\xi}=\left(\xi_{1}, \ldots, \xi_{n}\right)$ с независимыми в совокупности бернуллиевскими элементами, принимающими значения 1 и 0 с вероятностями $p_{0}$ и $q_{0}, p_{0}, q_{0}>0, p_{0}+q_{0}=1$. Вкрапление закрытой информации осуществляется путем случайного выбора в этом массиве $k$ позиций, $0<k \leqslant n$, и заменой на этих позициях исходных элементов другими бернуллиевскими элементами $\bar{\eta}=\left(\eta_{1}, \ldots, \eta_{k}\right)$, независимыми от исходных элементов и между собой и принимающих значения 1 и 0 с другими вероятностями $p_{1}$ и $q_{1}, p_{1}, q_{1}>0, p_{1}+q_{1}=1$. Эту операцию будем обозначать символом $\Delta$, таким образом, наблюдению доступен массив (последовательность) $\bar{\zeta}=\bar{\xi} \Delta \bar{\eta}$.

Основными задачами анализа такой модели являются

(1) проверка гипотезы $H_{0}$ об отсутствии вкраплений, то есть $H_{0}: k=0$, при альтернативе $H_{1}: k>0$ о наличии вкраплений, и

(2) оценивание числа $k$ вкраплений (при справедливости альтернативы $H_{1}$ ).

Параметрами описанной модели являются $p_{0}, p_{1}$ и $k$, и решение этих задач существенно зависит о априорных предположениях об этих параметрах. Ниже будут рассмотрены различные варианты конкретизации модели в терминах этих параметров.

\section{3. Статистический критерий}

Рассмотрим сначала случай, когда $p_{0}$ и $p_{1}$ известны, и будем считать, для определенности, что $\Delta p=p_{0}-p_{1}>0$. 
Для различения гипотез $H_{0}$ и $H_{1}$ в этом случае будем использовать тестовую статистику $\rho_{n}$, равную числу единиц в наблюденной последовательности $\bar{\zeta}=\bar{\xi} \Delta \bar{\eta}$, то есть

$$
\rho_{n}=\sum_{i=1}^{n} \zeta_{i}
$$

Очевидно, что при отсутствии вкраплений эта статистика имеет биномиальное распределение

$$
\mathscr{L}\left(\rho_{n} \mid H_{0}\right)=\operatorname{Bi}\left(n, p_{0}\right),
$$

в частности,

$$
\mathbf{E}\left(\rho_{n} \mid H_{0}\right)=n p_{0}, \quad \mathbf{D}\left(\rho_{n} \mid H_{0}\right)=n p_{0} q_{0} .
$$

Для изучения распределения статистики $\rho_{n}$ при альтернативе введем простую альтернативу $H_{1, k}$, при которой число вкраплений $k$ фиксировано. При этой альтернативе среди слагаемых в (1) присутствует $k$ элементов последовательности $\bar{\eta}$ (обозначим их сумму $|\eta|_{k}$ ) и $n-k$ элементов последовательности $\bar{\xi}$ (обозначим их сумму $|\xi|_{n-k}$ ). Таким образом, при альтернативе $H_{1, k}$ статистика $\rho_{n}$ имеет представление

$$
\rho_{n}=|\xi|_{n-k}+|\eta|_{k},
$$

причем слагаемые независимы и имеют соответственно биномиальные распределения $\operatorname{Bi}\left(n-k, p_{0}\right)$ и $\operatorname{Bi}\left(k, p_{1}\right)$. Следовательно,

$$
\mathscr{L}\left(\rho_{n} \mid H_{1, k}\right)=\operatorname{Bi}\left(n-k, p_{0}\right) * \operatorname{Bi}\left(k, p_{1}\right),
$$

где символ $*$ означает операцию свертки.

Из (4) и (5), в частности, следует, что

$$
\begin{aligned}
& \mathbf{E}\left(\rho_{n} \mid H_{1, k}\right)=(n-k) p_{0}+k p_{1}=n p_{0}-k \Delta p<n p_{0}, \\
& \mathbf{D}\left(\rho_{n} \mid H_{1, k}\right)=(n-k) p_{0} q_{0}+k p_{1} q_{1}=n p_{0} q_{0}-k\left(p_{0} q_{0}-p_{1} q_{1}\right) .
\end{aligned}
$$

С помощью соотношения (5) можно также вычислить и распределение статистики $\rho_{n}$ при альтернативе $H_{1, k}$ (функцию правдоподобия):

$$
\begin{aligned}
L_{n, k} & =\mathbf{P}\left\{\rho_{n}=s \mid H_{1, k}\right\}=\mathbf{P}\left\{|\xi|_{n-k}+|\eta|_{k}=s\right\} \\
& =\sum_{m=0}^{s \wedge k} \mathbf{P}\left\{|\eta|_{k}=m\right\} \mathbf{P}\left\{|\xi|_{n-k}=s-m\right\} \\
& =\sum_{m=0}^{s \wedge k}\left(\begin{array}{c}
k \\
m
\end{array}\right) p_{1}^{m} q_{1}^{k-m}\left(\begin{array}{l}
n-k \\
s-m
\end{array}\right) p_{0}^{s-m} q_{0}^{n-k-s+m} \\
& =p_{0}^{s} q_{0}^{n-k-s} q_{1}^{k} \sum_{m=0}^{s \wedge k}\left(\begin{array}{c}
k \\
m
\end{array}\right)\left(\begin{array}{l}
n-k \\
s-m
\end{array}\right) z^{m},
\end{aligned}
$$

где $s \wedge k=\min (s, k)$ и $z=p_{1} q_{0} /\left(q_{1} p_{0}\right)$.

При нулевой гипотезе функция правдоподобия есть

$$
L_{n, 0}(s)=\mathbf{P}\left\{\rho_{n}=s \mid H_{0}\right\}=\left(\begin{array}{l}
n \\
s
\end{array}\right) p_{0}^{s} q_{0}^{n-s},
$$


поэтому отношение правдоподобия равно

$$
\ell_{n, k}(s)=\frac{L_{n, k}(s)}{L_{n, 0}(s)}=\left(\frac{q_{1}}{q_{0}}\right)^{k} \sum_{m=0}^{s \wedge k} \frac{\left(\begin{array}{l}
k \\
m
\end{array}\right)\left(\begin{array}{l}
n-k \\
s-m
\end{array}\right)}{\left(\begin{array}{l}
n \\
s
\end{array}\right)} z^{m} .
$$

Эти результаты дают возможность построить критерий Неймана-Пирсона для различения гипотез $H_{0}$ и $H_{1, k}$ (см., например, [3]). Такой критерий задается критической областью

$$
\mathscr{X}_{1, \alpha}=\left\{s: \ell_{n, k}(s) \geqslant c_{\alpha}\right\},
$$

где критическая граница $c_{\alpha}$, отвечающая вероятности ошибки первого рода (уровню значимости) $\alpha$, определяется условием

$$
\mathbf{P}\left\{\mathscr{X}_{1, \alpha} \mid H_{0}\right\}=\mathbf{P}\left\{\ell_{n, k}\left(\rho_{n}\right) \geqslant c_{\alpha} \mid H_{0}\right\}=\alpha .
$$

Замечание 1. Отметим, что, с точностью до множителя $\left(q_{1} / q_{0}\right)^{k}$, отношение правдоподобия $\ell_{n, k}(s)$ есть производящая функция гипергеометрического распределения в точке $z=p_{1} q_{0} /\left(q_{1} p_{0}\right)$, которую, в свою очередь, можно записать через гипергеометрическую функцию

$$
F(a, b, c, z)=\sum_{m=0}^{\infty} \frac{(a)_{m}(b)_{m} z^{m}}{(c)_{m} m !}
$$

где $(a)_{m}=a(a+1) \ldots(a+m-1), m=1,2 \ldots,(a)_{0}=1$.

Именно,

$$
\frac{\left(\begin{array}{c}
k \\
m
\end{array}\right)\left(\begin{array}{c}
n-k \\
s-m
\end{array}\right)}{\left(\begin{array}{c}
n-k \\
s
\end{array}\right)}=\frac{(-k)_{m}(-s)_{m}}{m !(n-k-s+1)_{m}},
$$

поэтому

$$
\ell_{n, k}(s)=\left(\frac{q_{1}}{q_{0}}\right) \frac{\left(\begin{array}{c}
n-k \\
s
\end{array}\right)}{\left(\begin{array}{c}
n \\
s
\end{array}\right)} F(-k,-s, n-k-s+1 ; z) .
$$

Поскольку функция $R$ - хорошо изученная специальная функция с известным асимптотическим поведением с ростом ее аргументов [2], представление (12) может быть использовано при анализе асимптотического поведения отношения правдоподобия $\ell_{n, k}(s)$, когда $n, k \rightarrow \infty$.

\section{4. Асимптотический вариант критерия Неймана-Пирсона}

Из (8) видно, что точный расчет критерия (9)-(10) представляет собой трудную вычислительную задачу, поэтому будем рассматривать асимптотический вариант критерия при $n \rightarrow \infty$. Заметим, что асимптотический подход в данной проблематике вполне естествен, поскольку в действительности обычно имеют дело с большими массивами данных.

Введем нормированную статистику

$$
\rho_{n}^{*}=\frac{\rho_{n}-n p_{0}}{\sqrt{n p_{0} q_{0}}}
$$


которая при нулевой гипотезе $H_{0}$, согласно теореме Муавра-Лапласа, при $n \rightarrow \infty$ имеет в пределе нормальное распределение $\mathcal{N}(0,1)$. Из (6) следует, что

$$
\mathbf{E}\left(\rho_{n}^{*} \mid H_{1, k}\right)=-\frac{k \Delta p}{\sqrt{n p_{0} q_{0}}}, \quad \mathbf{D}\left(\rho_{n}^{*} \mid H_{1, k}\right)=1-\frac{k\left(p_{0} q_{0}-p_{1} q_{1}\right)}{n p_{0} q_{0}} .
$$

Согласно представлению (4), при альтернативе $H_{1, k}$ статистика $\rho_{n}^{*}$ также будет асимптотически нормальна с параметрами, приведенными в (14). Отсюда следует, что если число вкраплений $k$ таково, что $k / \sqrt{n} \rightarrow 0$, то гипотезы $H_{0}$ и $H_{1, k}$ асимптотически неразличимы, поскольку в этом случае $\mathscr{L}\left(\rho_{n}^{*} \mid H_{1, k}\right) \rightarrow \mathcal{N}(0,1)$, как и в случае нулевой гипотезы.

Статистика $\rho_{n}^{*}$ будет чувствительна к таким альтернативам $H_{1, k}$, при которых $k / \sqrt{n} \rightarrow \lambda>0$. Для этого случая

$$
\mathscr{L}\left(\rho_{n}^{*} \mid H_{1, k}\right) \rightarrow \mathcal{N}\left(-\lambda \Delta p / \sqrt{p_{0} q_{0}}, 1\right),
$$

и в асимптотике задача сводится к различению двух нормальных распределений $\mathcal{N}(0,1)$ и $\mathcal{N}\left(-\lambda \Delta p / \sqrt{p_{0} q_{0}}\right)$ по одному наблюдению над тестовой статистикой $\rho_{n}^{*}$.

Соответствующий критерий задается критической областью (см. с. 188 в [3])

$$
\mathscr{X}_{1, \alpha}^{*}=\left\{\rho_{n}^{*}<-t_{\alpha}\right\},
$$

где $\alpha$ - заданный уровень значимости, $\Phi(x)-$ функция стандартного нормального распределения и $\Phi\left(-t_{\alpha}\right)=\alpha$.

Мощность $W_{n, k}$ этого критерия, согласно (15), удовлетворяет асимптотическому соотношению

$$
W_{n, k}=\mathbf{P}\left\{\rho_{n}^{*}<-t_{\alpha} \mid H_{1, k}\right\} \rightarrow \Phi\left(\lambda \Delta p / \sqrt{p_{0} q_{0}}-t_{\alpha}\right) .
$$

Отметим, что предельная мощность (17) является монотонно возрастающей функцией параметра $\lambda$ (чем больше вкраплений, тем легче этот факт обнаруживается критерием (16)).

Замечание 2. Определенная в (16) критическая область $\mathscr{X}_{1, \alpha}^{*}$ не зависит от параметра $\lambda$, характеризующего альтернативу $H_{1, k}$, поэтому эта критическая область максимизирует мощность при любой допустимой альтернативе (в данном случае, согласно (14), все альтернативы являются левосторонними, то есть при любой из них распределение статистики $\rho_{n}^{*}$ сдвинуто влево по отношению к распределению $\mathcal{N}(0,1)$. Отсюда следует (см. с. 198 в [3]), что построенный критерий Неймана-Пирсона является равномерно наиболее мощным критерием проверки гипотезы $H_{0}$, состоящей в том, что $k=0$, при сложной альтернативе $H_{1}$, состоящей в том, что $k>0$.

Отметим также, что в случае $k / \sqrt{n} \rightarrow \infty$ альтернатива $H_{1, k}$ улавливается критерием (16) с вероятностью, стремящейся к единице, то есть против такой альтернативы этот критерий состоятелен.

Представим итог в виде следующего утверждения.

Теорема 1. В задаче различения гипотез $H_{0}$ и $H_{1}$ об отсутствии и наличии вкраплений соответственно существует асимптотически (при $n \rightarrow \infty)$ равномерно наиболее мощный критерий, задаваемый соотношениями (16), (13) и (1), асимптотическая мощность которого при альтернативах $H_{1, k}$, для которых $k=k(n)$ таково, что $k / \sqrt{n} \rightarrow \lambda>0$ (пороговый случай), удовлетворяет соотношению (17). При $k=o(\sqrt{n})$ альтернативь $H_{1, k}$ асимптотически этим критерием не отличаются от нулевой гипотезь, а против альтернатив $H_{1, k}$, для которых $k / \sqrt{n} \rightarrow \infty$, этот критерий состоятелен. 


\section{5. Схема серий}

Основной проблемой в стеганографии является скрытие от противника самого факта вкрапления передаваемой закрытой информации. С этих позиций для передающей стороны важно организовать процесс вкрапления так, чтобы гипотезы $H_{0}$ и $H_{1}$ были асимптотически при $n \rightarrow \infty$ неразличимы. Предыдущий анализ показывает, что если в рассматриваемой модели параметр вкрапления $p_{0}, p_{1}<p_{0}$, фиксирован, то сам факт вкрапления надежно маскируется лишь при числе вкраплений $k=o(\sqrt{n})$. Увеличить объем надежно маскируемой вкрапляемой информации (в рамках изучаемой модели) можно путем выбора параметра $p_{1}$, близкого к значению параметра $p_{0}$. В этом случае мы должны рассматривать схему серий, полагая $p_{1}=p_{1}(n) \rightarrow p_{0}$ при $n \rightarrow \infty$.

Итак, пусть при $n \rightarrow \infty$ разность $\Delta p=p_{0}-p_{1}=\Delta_{n} \rightarrow 0$. Тогда при близких (к гипотезе $H_{0}$ ) альтернативах $H_{1, k}$ тестовая статистика $\rho_{n}^{*}$ по-прежнему будет иметь асимптотически нормальное распределение

$$
\mathscr{L}\left(\rho_{n}^{*} \mid H_{1, k}\right) \sim \mathcal{N}\left(k \Delta_{n} / \sqrt{n p_{0} q_{0}}, 1\right)
$$

поскольку $p_{0} q_{0}-p_{1} q_{1}=\left(q_{0}-p_{0}\right) \Delta_{n}-\Delta_{n}^{2} \rightarrow 0$, в (14) $\mathbf{D}\left(\rho_{n}^{*} \mid H_{1, k}\right)=1+o(1)$ при всех $k \leqslant n$.

Из (18) следует, что факт вкрапления в этих условиях будет надежно маскироваться, если $k \Delta_{n} / \sqrt{n} \rightarrow 0$, то есть при $k=o\left(\sqrt{n} / \Delta_{n}\right)$.

Таким образом, чем меньше величина $\Delta_{n}=p_{0}-p_{1}$ (чем ближе $p_{1}=p_{1}(n)$ к $\left.p_{0}\right)$, тем большее число вкрапляемых символов $k$ можно передать, будучи уверенным в том, что факт вкрапления не будет обнаружен. В частности, при $\Delta \sim 1 / \sqrt{n}$ число вкраплений $k$ может быть велико, лишь бы выполнялось условие $k=o(n)$.

Пороговыми альтернативами в схеме серий являются альтернативы $H_{1, k}$, для которых $k \Delta_{n} / \sqrt{n} \rightarrow \delta>0$. Для таких альтернатив критерий (16) будет иметь асимптотическую мощность $\Phi\left(\delta / \sqrt{p_{0} q_{0}}-t_{\alpha}\right)$.

Итак, в схеме серий справедливо следующее утверждение.

Теорема 2. Если при $n \rightarrow \infty$ параметр вкрапления $p_{1}=p_{1}(n)=p_{0}-\Delta_{n}$, где $\Delta_{n} \rightarrow 0$, то при числе вкраплений $k=o\left(\sqrt{n} / \Delta_{n}\right)$ гипотезы $H_{0}$ и $H_{1}$ асимптотически неразличимьl, а при $k \Delta_{n} / \sqrt{n} \rightarrow \delta>0$ (в пороговом случае) мощность критерия (16) удовлетворяет асимптотическому соотночению

$$
W_{n, k}=\mathbf{P}\left\{\rho_{n}^{*},-t_{\alpha} \mid H_{1, k}\right\} \rightarrow \Phi\left(\delta / \sqrt{p_{0} q_{0}}-t_{\alpha}\right) .
$$

\section{6. Оценивание числа вкраплений}

Предыдущие результаты дают также возможность достаточно просто решить и задачу оценивания числа вкраплений $k$ по наблюдению статистики $\rho_{n}^{*}$. Именно, если имел место факт вкрапления (справедлива гипотеза $H_{1}=\bigcup_{k>0} H_{1, k}$ ), то из соотношения (6) следует, что статистика

$$
\hat{k}_{n}=\frac{n p_{0}-\rho_{n}}{\Delta p}
$$

удовлетворяет требованию несмещенности, то есть для любого $k>0$

$$
\mathbf{E}\left(\hat{k}_{n} \mid H_{1, k}\right)=\frac{1}{\Delta p}\left(n p_{0}-\mathbf{E}\left(\rho_{n} \mid H_{1, k}\right)\right)=k .
$$


Таким образом, $\hat{k}_{n}$ является несмещенной оценкой для $k$.

Более того, при $n \rightarrow \infty$ эта оценка является асимптотической оценкой максимального правдоподобия. Действительно, как показано в разделе 4, нормированная статистика $\rho_{n}^{*}$ (см. (13)) при любой гипотезе $H_{1, k}$ имеет асимптотически нормальное распределение $\mathcal{N}\left(-k \Delta p / \sqrt{n p_{0} q_{0}}, 1\right)$. Как известно (см., например, раздел 2.4 в [3]), для нормального распределения с неизвестным средним (в данном случае это среднее равно $-k \Delta p / \sqrt{n p_{0} q_{0}}$ ) оценкой максимального правдоподобия по одному наблюдению над соответствующей случайной величиной является ее наблюдаемая реализация, следовательно, в данном случае оценкой для $-k \Delta p / \sqrt{n p_{0} q_{0}}$ является $\rho_{n}^{*}$, и мы приходим к (20).

Вычислим дисперсию оценки $\hat{k}_{n}$. Из (6) получаем, что

$$
\begin{aligned}
\mathbf{D}\left(\hat{k}_{n} \mid H_{1, k}\right) & =\mathbf{D}\left(\rho_{n} / \Delta p \mid H_{1, k}\right)=\frac{1}{(\Delta p)^{2}}\left(n p_{0} q_{0}-k\left(p_{0} q_{0}-p_{1} q_{1}\right)\right) \\
& =\frac{n p_{0} q_{0}}{(\Delta p)^{2}}\left(1-\frac{k}{n}\left(1-\frac{p_{1} q_{1}}{p_{0} q_{0}}\right)\right) .
\end{aligned}
$$

Это соотношение показывает, что дисперсия оценки $\hat{k}_{n}$ с ростом $n$ неограниченно растет, то есть эта оценка не является состоятельной и поэтому практически непригодна.

Следовательно, в рассматриваемой задаче нужен другой подход, а именно, нужно оценивать не само число $k$ вкраплений, а, скажем, его долю $\lambda=k / n$. Этот новый параметр модели имеет в качестве области возможных значений отрезок $[0,1]$ и, как следует из раздела 5, чтобы реально этот отрезок не был исчезающе малым при больших значениях $n$, нужно рассматривать модель в схеме серий, когда $p_{1}=p_{1}(n) \rightarrow p_{0}$ при $n \rightarrow \infty$.

Итак, будем предполагать, что $n \rightarrow \infty$ и параметр вкраплений $p_{1}=p_{1}(n)$ имеет вид

$$
p_{1}=p_{0}+\frac{\tau}{\sqrt{n}},
$$

где $\tau$ - отрицательная постоянная. Тогда в этих обозначениях соотношение (18) принимает вид

$$
\mathscr{L}\left(\rho_{n}^{*} \mid H_{1, k}\right) \sim \mathcal{N}\left(\lambda \tau / \sqrt{p_{0} q_{0}}, 1\right) .
$$

Отсюда, как и выше, следует, что оценкой максимального правдоподобия параметра $\lambda=k / n$ в условиях (23) является статистика

$$
\hat{\lambda}_{n}=\frac{1}{\tau} \sqrt{p_{0} q_{0}} \rho_{n}^{*}=\frac{\rho_{n}-n p_{0}}{\tau \sqrt{n}} .
$$

Для нее выполняется асимптотическое соотношение

$$
\mathscr{L}\left(\hat{\lambda}_{n} \mid H_{1, k}\right) \sim \mathcal{N}\left(\lambda, p_{0} q_{0} / \tau^{2}\right),
$$

то есть она является асимптотически несмещенной, но по-прежнему не обладает свойством состоятельности: ее дисперсия не стремится к нулю при $n \rightarrow \infty$.

В то же время проведенный анализ показывает, что свойство состоятельности оценка (22) приобретает в ситуации, когда параметр $\tau=\tau(n) \rightarrow-\infty$ при $n \rightarrow \infty$, то есть при $-\tau=\left(p_{0}-p_{1}\right) \sqrt{n}=\sqrt{n} \Delta_{n} \rightarrow \infty$.

Таким образом, справедливо следующее утверждение.

Теорема 3. Если при $n \rightarrow \infty$ выполняются условия $\Delta_{n}=p_{0}-p_{1} \rightarrow 0, \sqrt{n} \Delta_{n} \rightarrow \infty$, то асимптотически несмещенной и состоятельной оченкой доли $\lambda=k / n$ числа вкраплений является статистика $\hat{\lambda}_{n}=\left(n p_{0}-\rho_{n}\right) /\left(n \Delta_{n}\right)=\hat{k}_{n} / n$, где $\hat{k}_{n}$ определено в (20). 
Более того, как следует из (21), эта оценка является в точности несмещенной, она в пределе имеет распределение $\mathcal{N}\left(\lambda, p_{0} q_{0} /\left(n \Delta_{n}^{2}\right)\right)$ и тем самым позволяет рассчитать асимптотический доверительный интервал для $\lambda$, при доверительном уровне $\gamma$ он имеет вид (см. с. 144 в [3])

$$
\left(\hat{\lambda}_{n} \pm c_{\gamma} \sqrt{p_{0} q_{0}} /\left(\sqrt{n} \Delta_{n}\right), \quad c_{\gamma}=\Phi^{-1}((1+\gamma) / 2) .\right.
$$

\section{7. Выводы}

С позиции обеспечения надежности сокрытия вкрапляемой информации (а это главная проблема стеганографии) процесс вкрапления (в рамках рассматриваемой модели) должен быть организован так, чтобы выполнялось единственное условие: $k \Delta_{n} / \sqrt{n} \rightarrow 0$ при $n \rightarrow \infty$, где $\Delta_{n}=p_{0}-p_{1} \rightarrow 0$.

В свою очередь, это условие может быть обеспечено двумя способами:

(1) $k=o(\sqrt{n})$, тогда параметр вкрапления $p_{1}$ может быть любым, но число вкрапляемых символов при этом незначительно,

(2) $\Delta_{n} \rightarrow 0$, то есть $p_{1}=p_{1}(n) \rightarrow p_{0}$, тогда для числа вкрапляемых символов возможности расширяются до границы $o\left(\sqrt{n} / \Delta_{n}\right)$, например, при $\Delta_{n} \sim 1 / \sqrt{n}$ число $k$ может быть любым вида $o(N)$.

\section{Список литературы}

1. Грибунин В. Г., Оков И. Н., Туринцев И. В., Цифровая стеганография. Солон-Пресс, Москва, 2002.

2. Бейтвен Г., Эрдейн А., Высиие трансиендентные функиии, 1. Наука, Москва, 1965.

3. Ивченко Г. И., Медведев Ю. И., Математическая статистика. Высшая школа, Москва, 1992.

Статья поступила 8.10.2008. 